\title{
Utilização das Ferramentas de Abordagem na Reintegração Familiar
}

\author{
The Use of The Approach Tools in Family Reintegration \\ Uso de las Herramientas de Enfoque en la Reintegración Familiar
}

Verônica Cardoso de Abreu ${ }^{1 *}$, Luis Paulo Morais Farias ${ }^{1}$, Carla Patrícia Martins Cardoso1, Samara Ferreira Gomes ${ }^{1}$, Rayssa de Luar Oliveira Dias Teixeira ${ }^{1}$, Jéssica Rejane Durães Soares ${ }^{1}$, Cláudio Wagnus Xavier Lopes Júnior ${ }^{1}$, Lorena Rodrigues Barbosa ${ }^{1}$, Mayara Karoline Silva Lacerda ${ }^{1}$, Vanessa Cristiane Araújo Oliveira1', Renata Francine Rodrigues de Oliveira1.

\section{RESUMO}

Detalhamento do caso/Estudo de caso: Paciente índice Fernanda, gênero feminino, 31 anos de idade, com despigmentação de retina, depressão, com ideação suicida, desempregada, solteira, residente em área de risco com cinco filhos, dos quais quatro passaram seis meses em uma Unidade de Acolhimento devido a negligências da mãe. Fez-se necessário intervir utilizando-se as ferramentas de abordagem familiar: Genograma, Ecomapa, Ciclo de vida Familiar, Firo e P.R.A.C.T.I.C.E junto aos integrantes após retorno dos filhos ao círculo familiar. Considerações finais: Uma vez que o papel da Estratégia Saúde da Família (ESF) é estimular a autonomia do sujeito para que este consiga formular soluções para as situações que interfiram no arranjo familiar, essa abordagem possibilitou a realização de um trabalho multiprofissional, com enfermeira, cirurgião-dentista e psicóloga, efetiva em parceria com os membros da família diante de um meio familiar exposto à vulnerabilidade social, possibilitando a melhoria das relações entre os membros e convívio entre os mesmos, reorganização da dinâmica familiar e, dessa forma, facilitando a resolução dos problemas.

Palavras-chave: Saúde da família, relações familiares, vulnerabilidade social.

\section{ABSTRACT}

Case Report: Patient index Fernanda, female, 31 years old, with retinal depigmentation, depression, with suicidal ideation, unemployed, residing at a risk area with five children, four of whom spent six months in a Shelter Unit due to her mother's negligence. It was necessary to intervene utilizing family approach tools: Genogram, Ecomap, Family Life Cycle, Firo and P.R.A.C.T.I.C.E. with members after returning of children to the family circle. Final considerations: Once the role of the Family Health Estrategy is stimulate the autonomy of the subject for them to formulate solutions for situation that interfere at the family arrangement, this approach made possible the accomplishment of an interdisciplinary work, with a nurse, a dental surgeon and a psychologist in partnership with the family exposed to social vulnerability, making possible improvement in the relation between members and conviviality among members, reorganization of the family dynamics and, thus, facilitating resolution of the problems.

Keywords: Family health, family relations, social vulnerability.

\section{RESUMEN}

Relato de caso: Pacientes indice Fernanda, mujer, 31 años, con despigmentación de la retina, depresión, ideación suicida, desempleada, soltera, que reside en un área de riesgo con cinco niños, cuatro de los cuales pasaron seis meses en una unidad de recepción debido a negligencia de la madre. Fue necesario intervenir utilizando las herramientas de enfoque familiares: Genogram, Ecomapa, Family Life Cycle, Firo y

${ }^{1}$ Universidade Estadual de Montes Claros (Unimontes), Montes Claros/MG. *E-mail: veronicaabreu101@hotmail.com 
P.R.A.C.T.I.C.E con los miembros después de regresar a los niños al círculo familiar. Consideraciones finales: Desde el papel de la Estrategia de Salud Familiar (FSE) y estimular la autonomía del sujeto para que pueda formular soluciones a situaciones que interfieren en el arreglo familiar, este enfoque hizo posible la realización de un trabajo multiprofesional, con una enfermera, un cirujano dental y un psicólogo, eficaz en asociación con los miembros de la familia frente a un entorno familiar expuesto a la vulnerabilidad social, permitiendo el mejoramiento de las relaciones entre los miembros y la interacción social entre ellos, reorganización de las dinámicas familiares y, por tanto, facilitando la resolución de problemas.

Palabras clave: Salud de la familia, relaciones familiares, vulnerabilidad social.

\section{INTRODUÇÃO}

Atenção Primária à Saúde (APS) é o conjunto de ações e serviços de saúde individuais, coletivos e familiares, realizados por equipe multiprofissional (BRASIL, 2017). Atualmente, o objeto do modelo assistencial das políticas de saúde pública está na família, tendo a Saúde da Família como a estratégia prioritária (BRASIL, 2017).

A Constituição Federal Brasileira de 1988 estabelece que a família seja a base da sociedade, recebendo especial proteção do estado, que deve assegurar assistência aos integrantes e coibir a violência (BRASIL, 1988).

A organização do sistema familiar é complexa e dinâmica, sofre mudanças à medida se transforma e pode influenciar internamente e externamente o bem-estar biopsicossocial de seus membros. Famílias expostas a dificuldades articulam-se através de mecanismos próprios de enfrentamento, baseados em crenças e valores culturalmente instalados (CHAPADEIRO CA et al., 2011).

Famílias são o foco da vigilância à saúde e do planejamento da assistência, além de ser o contexto do cuidado ao indivíduo (BRASIL, 2017). Faz-se necessário conhecer os integrantes e a situação social das famílias, para a identificação de demandas (FIGUEIREDO MHJS e MARTINS MMFS, 2010).

Para melhor entendimento das famílias, utilizam-se ferramentas de abordagem familiar, das quais, destacam-se como mais utilizadas na APS: Genograma, Ecomapa, Ciclo de vida Familiar, Firo (Fundamental Interpersonal Relations Orientation) e P.R.A.C.T.I.C.E. (Present Problem; Roles and Structure; Communication; Time in the Family Life Cycle; IIIness in Family Past and Present; Coping With Stress; Ecology) (SILVA MCBR et al., 2011).

\section{DETALHAMENTO DO CASO}

Trata-se de um estudo qualitativo, do tipo relato de caso. Seu desenvolvimento ocorreu no campo de atuação do Programa de Residência Multiprofissional em Saúde da Família, após a aprovação do Comitê de Ética em Pesquisa da Universidade Estadual de Montes Claros (Unimontes), sob parecer número 572.244/2014. A confidencialidade dos participantes foi resguardada utilizando-se nomes fictícios.

Uma assistente social e a psicóloga de uma Unidade de Acolhimento compareceram à Estratégia Saúde da Família (ESF) relatando que quatro crianças, recém-saídas da Unidade de Acolhimento, estavam regressando ao lar, após quatro meses vividos em uma unidade de abrigo. A equipe multiprofissional acolheu a demanda e, após visitas domiciliares (VDO), verificou-se a necessidade de abordagem familiar, uma vez que a família não tinha estrutura organizacional e dessa forma, corria-se o risco das crianças, por ordem judicial, voltarem à Unidade de Acolhimento.

A paciente índice Fernanda, 31 anos, possui ensino fundamental incompleto ( $3^{\text {a }}$ série), solteira, desempregada, cinco filhos, Jaqueline (16 anos), Rafaela (14 anos), João (11 anos), Michele (9 anos) e Bianca (6 anos), além disso, não possui companheiro. A residência da família é cedida pelos vizinhos e localiza-se em área de risco por tráfico de drogas e condições precárias de infraestrutura. A renda familiar 
advém de programas sociais, como o Benefício Assistencial à Pessoa com Deficiência (BPC) e Bolsa Família. A família também é acompanhada pelo Centro de Referência de Assistência Social (CRAS), recebendo frutas e verduras para complementar a alimentação.

Fernanda nasceu na cidade Jaíba/MG mas com a perda precoce dos pais foi criada pela avó. Aos três dias de vida perdeu a mãe (Antônia), vítima de assassinato (envenenada pela amante do pai). Após três meses da morte de sua mãe, o pai foi assassinado pela mesma autora. Com um ano de idade, Fernanda passou a residir em Montes Claros com a avó e seus tios, inicialmente, como moradores de rua.

Dos cinco filhos, dois (terceiro e o quarto filhos) são do mesmo relacionamento e os demais de outros relacionamentos temporários, sendo estes usuários de drogas e traficantes. Fernanda concebeu a primeira filha (Jaqueline) aos 13 anos de idade, após abuso sexual. Antes da sua última gestação, Fernanda abortou espontaneamente, por causa desconhecida, além disso, a gravidez da filha Bianca se deu mesmo após laqueadura.

Em fevereiro de 2018, Jaqueline, João, Michele e Bianca foram abrigados em uma Unidade de Acolhimento, devido a negligências da responsável, e retornaram para casa 4 meses depois. Esse reencontro desencadeou instabilidade à família, resultando em papeis intrafamiliares mal definidos.

Fernanda possui retinose despigmentar, doença crônica degenerativa e irreversível, com risco de cegueira. Apresenta baixa acuidade visual, necessitando de ajuda para realizar atividades domésticas, principalmente à noite.

No passado realizava faxinas como ofício, mas atualmente não é mais apta. Nas consultas e visitas domiciliares, refere medo de ficar cega e não conseguir cuidar dos filhos. A paciente apresenta também depressão e ideação suicida.

Durante as VDO, atendimentos individuais, contato com a escola e o CRAS, observou-se que as duas filhas mais velhas apresentaram dificuldades escolares e relacionamentos amorosos perigosos com traficantes de drogas.

Durante as VDO foram aplicadas as ferramentas de abordagem familiar: Genograma, Ecomapa, Ciclo de Vida Familiar, Firo e P.R.A.C.T.I.C.E. para o maior conhecimento da família. Por fim, foi realizada a Conferência Familiar para realização de intervenção e definição de responsabilidades intrafamiliares.

\section{DISCUSSÃO}

A unidade famíliar caracteriza-se pelas inter-relações estabelecidas entre os seus membros, em um meio com características exclusivas de organização, estrutura e funcionalidade (FIGUEIREDO MHJS; MARTINS MMFS., 2010).

O genograma é uma ferramenta eficiente para se obter informações da constituição familiar. Consiste na representação gráfica da família, permitindo uma clara visualização da sua estrutura interna, o que possibilita aos membros da família visualizarem suas patologias e o desenvolvimento dessas (LACERDA MKS et al., 2017). Nele são retratados os diversos membros familiares, o tipo de relacionamento entre eles e as principais morbidades (FIGUEIREDO MHJS e MARTINS MMFS, 2010; CHAPADEIRO CA et al., 2011).

Acrescentando-se informações ao genograma, o ecomapa é um diagrama que representa a visão geral da situação da família, retratando as relações de educação ou conflitos entre ela e o mundo. A família é colocada dentro de um círculo central e fazem-se círculos externos representando pessoas, órgãos ou instituições no contexto familiar. É utilizada para conhecimento da rede social da família, compreendendo a relação da família e o meio que a cerca (BAIA RSM et al., 2014).

A família da paciente índice é numerosa e cheia de conflitos (Figura 1). A mãe não consegue exercer seu papel de autoridade sobre os filhos e estes não a respeitam. Com essa ferramenta foi possível observar as interações intrafamiliares, as relações afetivas entre os membros e patologias associadas, tornando uma ferramenta necessária para a realização das ações de intervenção (LACERDA MKS et al., 2017). 
Figura 1 - Genograma da paciente índice Fernanda, março de 2019.
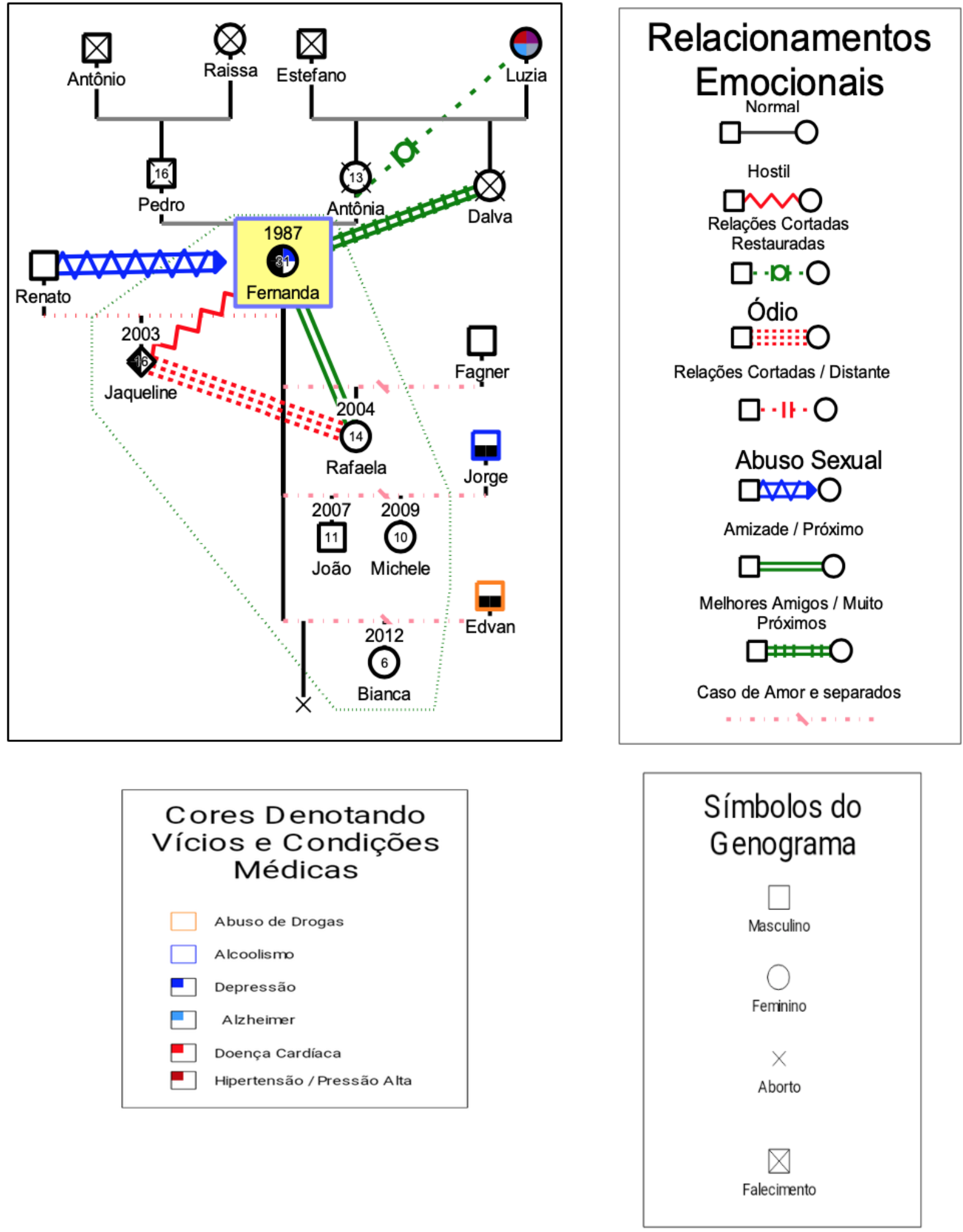

Fonte: Elaborado pelos autores, 2019.

A análise do ecomapa (Figura 2), demostra que a família possui boa relação com a equipe de saúde, observando o vínculo. Entretanto, os seus membros não são assíduos nas consultas agendadas e abandonam tratamentos já iniciados. 
Figura 2 - Ecomapa da família estudada, março de 2019.

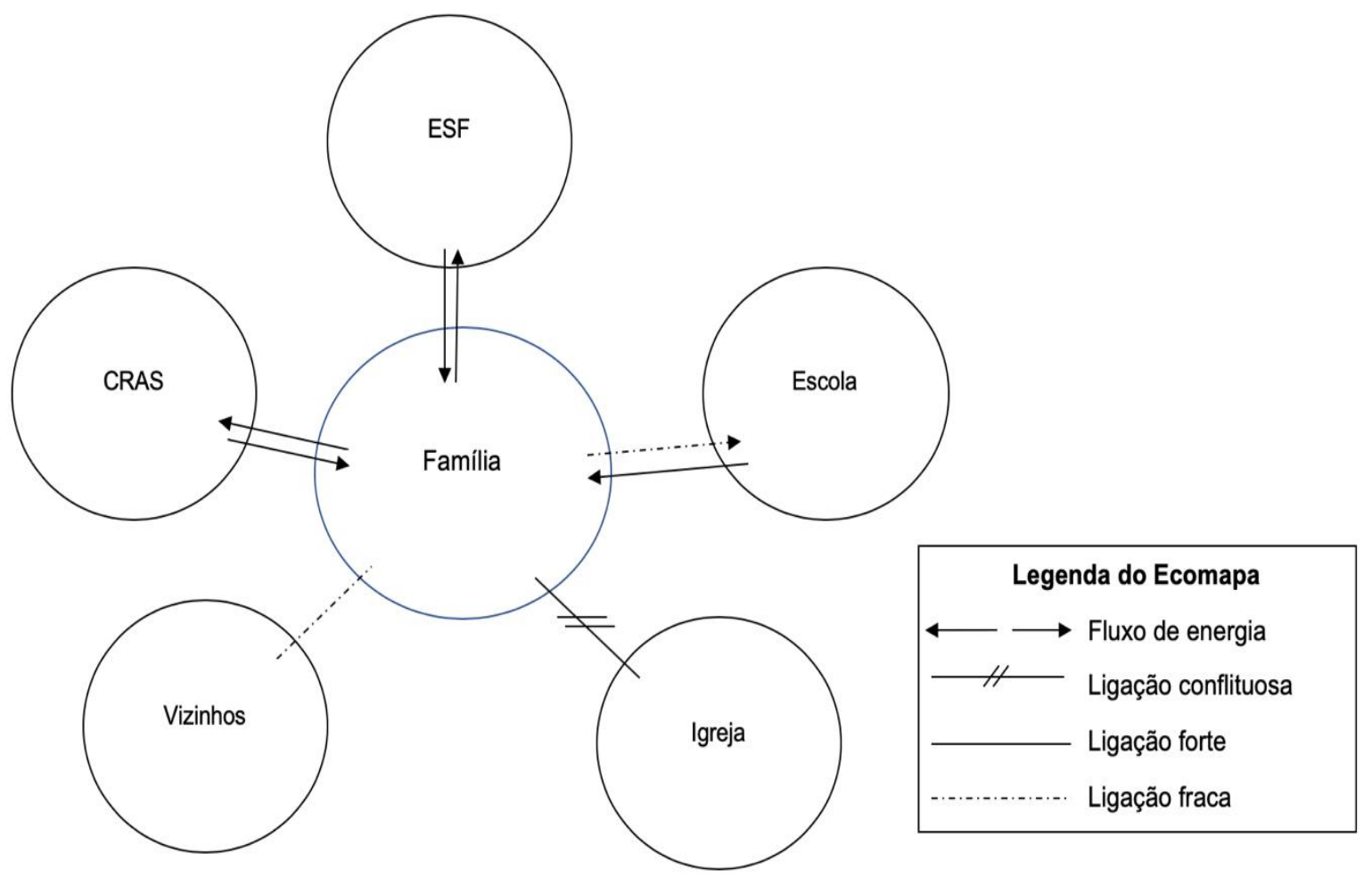

Fonte: Elaborado pelos autores, 2019.

A Igreja Adventista ajudou a família quando moravam na rua. Atualmente, eles frequentam a Igreja, porém, houve uma ruptura devido a conflitos e desentendimentos passados. Há ainda vínculo forte com o CRAS, no qual a família consegue apoio e benefícios. Possuem vínculo fraco com os vizinhos e moderado com a escola.

Outra ferramenta utilizada é o ciclo de vida familiar, que é dividida em oito estágios de desenvolvimento, na qual cada estágio é caracterizado por papéis e tarefas específicas. Descrevendo cada estágio: estágio I, fase em que se inicia a vida em casal; estágio II, meio familiar com filhos pequenos; estágio III, famílias com pré-escolares; estágio IV, famílias com filhos em idade escolar; estágio V, famílias com adolescentes; estágio VI, famílias com os filhos em partida; estágio VII, casais de meia idade e estágio VIII - famílias em estágio tardio de vida (ALVES AP et al., 2015).

Considerando o Ciclo de Vida Familiar, a família em estudo apresenta-se no estágio V. Neste estágio há necessidade que exista equilíbrio entre liberdade e responsabilidade, além de bastante interação entre os membros. É necessária uma flexibilização dos pais e o aumento da autonomia dos filhos (ALVES AP et al., 2015).

O modelo FIRO consiste no acrônimo: Orientações Fundamentais nas Relações Interpessoais, traduzido da língua inglesa que representa Fundamental Interpersonal Relations Orientations. Seu intuito destina-se a compreender o funcionamento da família e avaliar os sentimentos dos seus membros, na convivência das relações cotidianas (CHAPADEIRO CA et al., 2011).

As relações familiares podem ser representadas por três dimensões: a inclusão, que refere-se à interação dentro da família para sua vinculação e organização; o controle, que reflete o poder dentro da família e intimidade, ou seja, as interações familiares, relacionado às trocas interpessoais ou como os sentimentos são compartilhados (SANTOS JAD et al., 2016). 
O modelo FIRO é útil para avaliar a reintegração no convívio familiar após a experiência na Unidade de Acolhimento. Avaliando-se a categoria "inclusão", observa-se que a filha Jaqueline tem dificuldade em se adequar à rotina da casa, depois da reintegração, criando relação de atrito com Fernanda. Observa-se na categoria "controle", o qual remete ao exercício de poder na família, a inexistência de um membro responsável pela organização e com poder de autoridade. Na categoria "intimidade", percebe-se que há boa relação de cumplicidade da mãe Fernanda com a filha Rafaela.

Acrescentando-se ao entendimento das relações familiares, o instrumento P.R.A.C.T.I.C.E permite a avaliação do funcionamento da família. Representa o acrônimo, em português: Problema; Papeis e Estrutura; Afeto; Comunicação; Tempo no Ciclo de Vida; Doenças na Família; lidando com o Estresse; Meio Ambiente (DITTERICH RG et al., 2009).

- Problems (problemas apresentados): Desorganização da residência e desrespeito à autoridade materna. Os trabalhos domésticos não são igualitariamente divididos entre os membros, uma vez que a mãe necessita de auxílio devido aos seus problemas de visão.

- Roles (papéis): Os papeis intrafamiliares não são bem definidos, uma vez que a mãe não consegue gerenciar a família, nem definir regras para os membros adolescentes.

- Affect (afeto): Jaqueline, que foi concebida após abuso sexual, possui relação hostil com Fernanda, sobretudo quando a mãe interfere no seu relacionamento amoroso, o que já provocou, segundo relatos de Fernanda, agressão física e tentativa de homicídio por parte da filha.

- Communication (comunicação): Fernanda é a responsável pela comunicação entre os serviços de saúde e os demais membros da família.

- Time in Life (tempo no ciclo de vida): A família encontra-se no estágio $\mathrm{V}$, com filhos adolescentes, percebendo-se dificuldade da mãe em exercer o seu papel de chefe de família.

- IIIness (doenças no passado e no presente): Fernanda possui retinose despigmentar, doença crônica que leva à perda da visão. João possui vitiligo, doença da pele, não infectocontagiosa.

- Copingwith stress (lidando com o estresse): Devido à desorganização dos papéis intrafamiliares, a família possui dificuldade de enfrentamento dos problemas, os quais se intensificaram com o retorno das crianças da Unidade de Acolhimento.

- Enviroment/Ecology (ecologia/meio ambiente): A área onde se localiza às beiras de um córrego, desprovida de cuidados básicos de estrutura e limpeza.

Ao término da aplicação das ferramentas, foram elencados os problemas e, no intuito de solucioná-los, juntamente com a participação familiar, foi planejada e realizada a conferência familiar. A Conferência familiar é uma forma de intervir junto aos membros da família para que eles mesmos se articulem e busquem a resolução dos problemas, conduzida por profissionais que buscam a compreensão dos problemas da família (SILVA DR, 2009). Foi então realizada a conferência familiar com de todos os membros da família, sendo a enfermeira a moderadora do encontro, além da presença do cirurgião-dentista da ESF. Inicialmente foi exposto à família os problemas: sobrecarga de afazeres domésticos da mãe; falta de compreensão por parte dos filhos em relação à depressão da mãe; falta de autoridade da mãe e relação conflituosa entre os membros.

Durante a conferência familiar os familiares falaram suas opiniões, condição necessária para a criação de vínculo familiar e possibilitar a execução de possíveis intervenções (LACERDA MKS et al., 2017).

Fernanda mencionou que não se sentia bem, estava triste e com vontade de ficar isolada. Às vezes tem vontade de morrer e tem medo de dormir e acordar cega, sem ninguém para cuidar dos filhos. Segundo a mesma, os filhos acordam tarde, não auxiliam nas atividades domiciliares e há muitos conflitos. Menciona dificuldade financeira para o sustento da casa. As filhas concordam que é necessária a divisão preestabelecida das tarefas domésticas para a diminuição dos conflitos. Jaqueline e Rafaela referem desejo de trabalhar para terem seu próprio dinheiro. Concluem que a família precisa de ajuda. 
Utilizando-se das ferramentas de abordagem familiar e a partir do vínculo criado junto aos membros, foi possível realizar intervenções necessárias. Inicialmente, foi ofertado apoio psicológico à Fernanda, devido à depressão e ideação suicida. Em seguida, toda família foi assistida pela equipe multidisciplinar (psicóloga, enfermeira, médica e cirurgião-dentista). Fernanda, Jaqueline e Rafaela realizam controle de natalidade e participam das reuniões de Planejamento Familiar. Jaqueline e Rafaela foram cadastradas no programa Jovem Aprendiz e receberam orientações sobre comportamento diante de uma entrevista de emprego.

Foi proposta a organização intrafamiliar de afazeres domésticos. Através de um quadro distribuiu-se os dias e as atividades de cada integrante, de acordo com a disponibilidade. Pactuou-se entre os membros responsabilidades para a organização da casa, sendo está uma das exigências da equipe do CRAS após o retorno das crianças.

A institucionalização é uma medida de proteção utilizada quando crianças e adolescentes se encontram em risco, tendo seus direitos violados. Devendo esta medida ser provisória, o retorno deve ser viabilizado quando a família apresentar condições favoráveis, pois é direito fundamental de toda criança e adolescente conviver em família e em comunidade (Estatuto da Criança e do Adolescente [ECA], 1990). Em concordância com o ECA, a decisão pela institucionalização das crianças se deu em função da desorganização intrafamiliar e negligência de cuidado por parte da mãe. O retorno ocorreu em curto período de tempo, quatro meses.

No Brasil ainda existe uma carência de literatura a respeito da reintegração familiar, comparado a outros países como Estados Unidos e países da Europa (SIQUEIRA AC et al., 2010). No presente estudo observase que a responsabilidade do acompanhamento da reintegração familiar foi repassada a ESF, diferente do que é recomendado pelo ECA, que atribui ao abrigo a tarefa de reinserção familiar. Dessa maneira, como é encorajado por Siqueira AC et al. (2010), uma política de reinserção familiar poderia ser criada, com uma equipe formada por assistentes sociais e psicólogos, em parceria com instituições de saúde que já desenvolvem seu trabalho junto a famílias, como a ESF.

Ressalta-se o papel da ESF é fazer com que o sujeito consiga criar soluções para conflitos existentes em arranjos familiares (PRATA LL et al., 2013). A abordagem familiar permitiu uma atuação multiprofissional efetiva em parceria com os membros da família, tornando-se possível a melhoria da relação e convívio entre os mesmos, reorganização da dinâmica familiar e facilitando, assim, a resolução dos problemas.

\section{REFERÊNCIAS}

1. ALVES AP et al. Ferramentas de abordagem familiar na Estratégia Saúde da Família: relato de caso da Equipe Vila Greyce em Montes Claros, Minas Gerais, Brasil. Revista EFDeportes, 2015; 19(202):1-8.

2. BAIA RSM et al. Enfermeiras e famílias: guia para avaliação e intervenção na família. 5ª ed. São Paulo (SP): Roca; 2012. Revista Rene, 2014; 15(5): 904-905.

3. BRASIL. Constituição (1988). Constituição da República Federativa do Brasil. Brasília, DF: Senado Federal: Centro Gráfico, 1988. 292 p.

4. BRASIL. Ministério da Saúde. Portaria n 2.436 de 21 de setembro de 2017. Brasília: Diário Oficial da República Federativa do Brasil; 2017.

5. CHAPADEIRO CA et al. A família como foco da atenção à saúde. Belo Horizonte: Nescon/UFMG [Internet]. 2011; 96p.

6. DITTERICH, RG et al. As ferramentas de trabalho com famílias utilizadas pelas equipes de saúde da família de Curitiba, PR. Revista Saúde e Sociedade, 2009; 18(3): 515-524.

7. Estatuto da Criança e do Adolescente. (1990). Diário Oficial da União. Lei nº 8.069, de 13 de julho de 1990. Brasília, DF.

8. FIGUEIREDO MHJS; MARTINS MMFS. Avaliação familiar: do Modelo Calgary de Avaliação da Família aos focos da prática de enfermagem. Ciência Cuidado e Saúde, 2010; 9(3): 552-559.

10. LACERDA MKS et al. Ferramentas de abordagem familiar: estudo de uma família cadastrada em uma equipe de estratégia saúde da família em Montes Claros, Minas Gerais, Brasil. Revista de Iniciação Científica Vale do Rio Verde, 2017; 7(1): 25-34.

11. OLIVEIRA RG; MARCON SS. Trabalhar com famílias no Programa de Saúde da Família: a prática do enfermeiro em Maringá-Paraná. Revista Escola de Enfermagem da USP, 2007; 41(1): 65-72.

12. PRATA LL. Família e Cuidado sob os Olhares de uma Equipe de Saúde da Família de São Carlos, SP. Revista APS, 2013; 16(3): 250-257.

13. SANTOS JAD et al. Ferramenta de abordagem familiar na atenção básica: um relato de caso. Journal of the Health Sciences Institute, 2016; 34(4): 249-252.

14. SILVA DR. Famílias e situações de luto. In: OSORIO, L. C.; VALLE, M. E. P. Manual de terapia familiar, 2009; 376-398.

15. SILVA MCBR et al. A abordagem à família na Estratégia Saúde da Família: uma revisão integrativa da literatura. Revista da Escola de Enfermagem da USP, 2011; 45(5): 1250-1255.

16. SIQUEIRA AC et al. Processo de reinserção familiar: estudo de casos de adolescentes que viveram em instituição de abrigo. Estudos de Psicologia, 2010; 15(1): 7-15. 